\title{
Q-balls in Underground Experiments
}

\author{
M. Ouchrif a $*$ \\ aDipartimento di Fisica dell'Università di Bologna \\ and INFN Sezione di Bologna, \\ Viale Berti Pichat 6/2, I-40127, Italy. \\ and \\ Université Mohamed Premier, Faculté des Sciences, \\ Département de Physique, L.P.T.P. \\ B.P: 52460000 Oujda, Morocco
}

In this paper we present some features of Q-balls and we discuss their interactions with matter, and their energy losses in the Earth, for a large range of velocities. These calculations are used to compute the fractional geometrical acceptance of the MACRO detector. Furthermore a systematic analysis of the energy losses of Q-balls in three types of detectors is investigated. More specifically we have computed the light yield in liquid scintillators, the ionization in streamer tubes and the Restricted Energy Loss in the CR39 nuclear track detectors.

\section{INTRODUCTION}

Dark Matter (DM) is one of the most intriguing problems in particle physics and cosmology. Several types of stable particles hypothesized in theories beyond the Standard Model of particle physics have been considered as candidates for DM. One example of such particles is the lightest supersymmetric particle (LSP) coming from a supersymmetric extension of the Standard Model [1].

In theories where scalar fields, carry a conserved global quantum number, $Q$, there may exist non-topological solitons which are stabilized by global charge conservation. These particles are spherically symmetric and for large values of $Q$ their masses and volumes grow linearly with $Q$. Thus they act like homogenous balls of ordinary matter, with $Q$ playing the role of particle number; Coleman called this type of matter Q-balls [2].

The conditions for the existence of absolutely stable Q-balls are satisfied in supersymmetric theories with low energy supersymmetry breaking. According to 1 abelian non-topological solitons with Baryon and/or Lepton quantum numbers naturally appear in the spectrum of the Mini-

\footnotetext{
*e-mail: ouchrif@bo.infn.it
}

mal Supersymmetric Standard Model. The role of conserved quantum number is played by the baryon number. The same reasoning applies to sleptons for the lepton number and also to scalar Higgs particles. Q-balls can thus be considered like coherent states of squarks, sleptons and Higgs fields. Under certain assumptions about the internal self interaction of these particles and field the Q-balls are absolutely stable [4.

In this note we recall the main physical and astrophysical properties of these particles, the interaction of Q-balls with matter and their energy losses in matter, and the possibility of traversing the Earth to reach the MACRO detector [5]. We neglect the possibility of :

( $i)$ electromagnetic radiation emitted by Q-balls of high $\beta$.

(ii) strong interaction of Q-balls in the upper atmosphere capable of destroying the Q-ball [1]; this last point deserves further investigations.

\section{PROPERTIES OF Q-BALLS}

Q-balls could have been produced in the Early Universe, and could contribute to the DM. Several mechanisms could have lead to the formation of Q-balls in the Early Universe. They may have been created in the course of a phase transition, 
which is sometime called "solitogenesis", or they could have been produced via fusion in processes reminiscent of the big bang nucleosynthesis, wich have been called "solitosynthesis"; small Q-balls can be pair-produced in high energy collisions [3].

The astrophysical consequenses of Q-balls in many ways resemble those of strange quark matter, "nuclearites"; the peculiarity of Q-balls is that their mass grows as $Q^{3 / 4}$, while for nuclearites the mass grows linearly with baryon number [6].

The Q-ball mass and size are related to its baryon number [7]. For a supersymetic potential $U(\phi) \sim M_{S}^{4}=$ constant for large scalar $\phi$, the Q-ball mass $M$ and radius $R$ are given by [7].

$M=\frac{4 \pi \sqrt{2}}{3} M_{S} Q^{3 / 4}$

$R=\frac{1}{\sqrt{2}} M_{S}^{-1} Q^{1 / 4}$

The parameter $M_{S}$ is the energy scale of the SUSY breaking symmetry. A stability condition was found in ref. [7]: the Q-ball mass $M$ is related to the nucleon mass $M_{N}$ by

$M \leq Q M_{N}$

From Eq. 1 and Eq. 3 one has the stability constraint [7]:

$Q \geq 1.6 \times 10^{15}\left(\frac{M_{S}}{1 \mathrm{TeV}}\right)^{4}$

In Fig. 1 the allowed region for stable Q-balls is indicated.

Q-balls are expected to concentrate in galactic halos and to move with the typical galactic velocity $v=\beta c \sim 10^{-3} c$. Assuming that Qballs constitute the cold galactic dark matter with $\rho_{D M} \sim 0.3 \mathrm{GeV} / \mathrm{cm}^{3}$, their number density is [7]

$N_{Q} \sim \frac{\rho_{D M}}{M} \sim 5 \times 10^{-5} Q^{-3 / 4}\left(\frac{1 T e V}{M_{S}}\right) \mathrm{cm}^{-3}(5)$

The corresponding flux is [7]

$\phi \sim 10^{2} Q^{-3 / 4}\left(\frac{M_{S}}{1 T e V}\right)^{-1} \mathrm{~cm}^{-2} s^{-1} s r^{-1}$

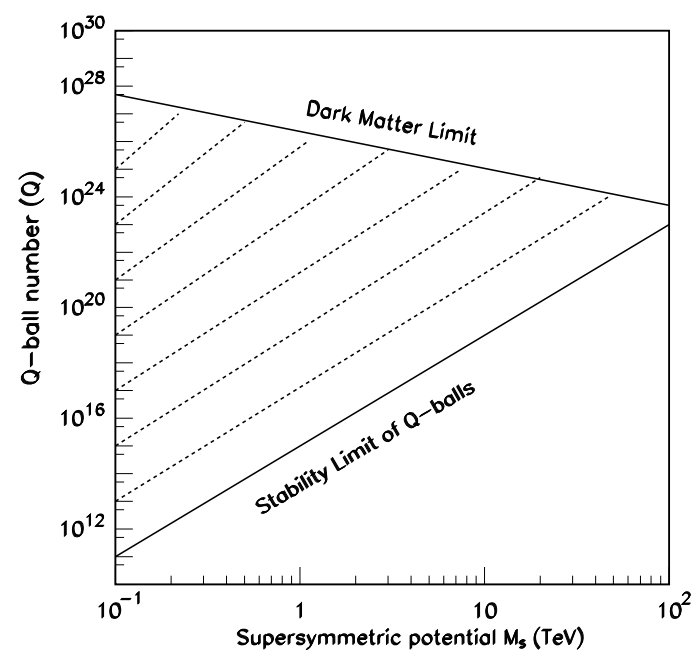

Figure 1. Q-ball number versus the supersymmetry energy scale $M_{S}$ for Q-balls. The shaded allowed region is delimited by the Q-ball stability limit (Eq. 4) and by the DM Limit, Eq. 6.

If we assume that $\beta_{Q-\text { ball }} \sim 10^{-3}, \rho_{D M} \sim$ $0.3 \mathrm{GeV} / \mathrm{cm}^{3}, M_{S}=1 \mathrm{TeV}$, the Q-ball flux cannot be greater that $\sim 4 \times \frac{10^{-19}}{M_{Q}} \mathrm{~cm}^{-2} \mathrm{~s}^{-1} \mathrm{sr}^{-1}$ $\left(M_{Q}\right.$ in g). Q-balls are expected to be distributed uniformly in our part of the galaxy and there should not be enhancements in the solar system, as for example a cloud of Q-balls around the sun.

Q-balls can be classified in two classes: SECS (Supersymmetric Electrically Charged Solitons) and SENS (Supersymmetric Electrically Neutral Solitons). The interaction of Q-balls with matter and their detection differ drastically for SECS or SENS.

\section{INTERACTION WITH MATTER}

\subsection{Interaction with matter of Q-balls type SECS}

SECS are Q-balls with a net positive electric charge in the interior. The charge of SECS originates from the unequal rate of absorption in the condensate of quarks (squarks) and electrons (se- 
lectrons). This positive electric charge is neutralized by a surrounding cloud of electrons. The positive charge interacts via elastic or quasi elastic collisions. The positive electric charge can vary from one to several tens; the cross section is the Bohr cross section for Q-ball hydrogeninteraction [

$\sigma=\pi a_{0}^{2} \sim 10^{-16} \mathrm{~cm}^{2}$

Where $a_{0}$ is the Bohr radius. The formula is valid for $R \geq a_{0}$, which happens for $Q \geq 2.7 \times\left(\frac{M_{S}}{1 T e V}\right)^{4}$.

The main energy losses [5] of SECS passing throuth matter with velocities in the range $10^{-4}<\beta<10^{-2}$ is due to two contributions: the energy losses due to $(i)$ the interaction of the SECS core with the nuclei (nuclear contribution) and, (ii) with the electrons of the traversed medium (electronic contribution). The total energy loss is the sum of the two contributions.

SECS could cause the catalysis of proton decay, but only if they are large and have large velocities [8]. The possibility that SECS can cause the catalysis of proton decay does not concern our range of interest for velocities, masses and radii of SECS.

Electronic losses of SECS: The electronic contribution to the energy loss of SECS is calculated with the following formula [5]

$\frac{d E}{d x}=\frac{8 \pi a_{0} e^{2} \beta}{\alpha} \frac{Z_{1}^{7 / 6} N_{e}}{\left(Z_{1}^{2 / 3}+Z_{2}^{2 / 3}\right)^{3 / 2}} \quad$ for $Z_{1} \geq 1(8)$

where $\alpha$ is the fine structure constant, $\beta=v / c$, $Z_{1}$ is the positive core charge of SECS, $Z_{2}$ is the atomic number of the medium and $N_{e}$ is the density of electrons in the medium. Electronic losses dominate for $\beta>10^{-4}$.

Nuclear losses of SECS: The nuclear contribution to the energy loss of SECS is due to the interaction of the SECS positive core with the nuclei of the medium and it is given by [5]

$\frac{d E}{d x}=\frac{\pi a^{2} \gamma N E}{\epsilon} S_{n}(\epsilon)$

where

$S_{n}(\epsilon) \simeq \frac{0.56 \log (1.2 \epsilon)}{1.2 \epsilon-(1.2 \epsilon)^{-0.63}}, \quad \epsilon=\frac{a M_{2} E}{Z_{1} Z_{2} e^{2} M_{1}}(10)$

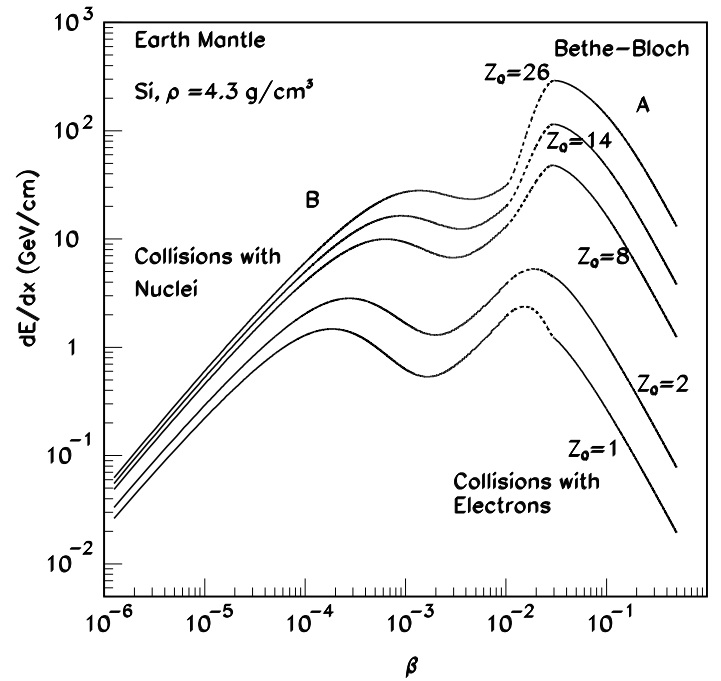

Figure 2. Energy losses of SECS versus $\beta$ in the Earth Mantle. $Z$ is the electric charge of the Qball core.

and

$a=\frac{0.885 a_{0}}{\left(\sqrt{Z_{1}}+\sqrt{Z_{2}}\right)^{2 / 3}}, \quad \gamma=\frac{4 M_{2}}{M_{1}}$

$M_{1}=M$ is the mass of the incident Q-ball; $M_{2}$ is the mass of the target nuclei; $Z_{1} e$ and $Z_{2} e$ are their electric charges; we assume that $M_{1}>M_{2}$. Nuclear losses dominates for $\beta \leq 10^{-4}$.

The energy losses of SECS in the earth mantle and earth core:

The energy losses of SECS in the earth mantle and earth core have been computed for different $\beta$-regions and for different charges of the $\mathrm{Q}$-ball core, using the same general procedures used in the past for computing the energy losses in the earth of magnetic monopoles and nuclearites [5].

In Fig. 2 is presented the energy losses of Qballs type SECS in the Earth mantle.

\subsection{Interaction of Q-balls type SENS}

The Q-ball interior of SENS is characterized by a large Vacuum Expectation Value (VEV) of certain squarks, and may be sleptons and Higgs 
fields [ [ 4 . The $S U(3)_{c}$ symmetry is broken and deconfinement takes place inside the Q-ball. If a nucleon enters this region of deconfinement, it dissociates into three quarks, some of which may then become absorbed in the condensate via the reaction [7].

$q q \rightarrow \tilde{q} \tilde{q}$

In practice the reaction looks like

$(Q)+$ Nucleon $\rightarrow(Q+1)+$ pions

and sometimes as

$(Q)+$ Nucleon $\rightarrow(Q+1)+$ Kaons

If it is assumed that the energy released in (13) is the same as in typical hadronic processes (about $1 \mathrm{GeV}$ per nucleon), this energy is carried by 2 or 3 pions (or 2 kaons). The cross section for reactions (13) and (14) is determined by the Q-ball radius $R$ []

$\sigma \sim 6 \times 10^{-34} Q^{1 / 2}\left(\frac{1 T e V}{M_{S}}\right)^{2} \mathrm{~cm}^{2}$

The corresponding mean free path $\lambda$ is

$\lambda=\frac{1}{\sigma N}$

According to References [5-8] the energy loss of SENS moving with velocities in the range $10^{-4}<$ $\beta<10^{-2}$ is constant and is given by

$\frac{d E}{d x} \sim \frac{\zeta}{\lambda}=\zeta N 10^{-34} Q^{1 / 2}\left(\frac{1 T e V}{M_{S}}\right)^{2} \mathrm{~cm}^{2}$

where $N$ is the number of atoms $/ \mathrm{cm}^{3}$ and $\zeta$ is the energy released in the decay. The energy loss of SENS is due to the energy released from the absorbed nucleon mass. SENS lose a very small fraction of their kinetic energy and are able to traverse the Earth without attenuation for all masses of our interest.

\section{ENERGY LOSSES IN DETECTORS}

\subsection{Light Yield of Q-balls type SECS}

For SECS we distinguish two contributions to the Light Yield in scintillators: the primary Light Yield and the secondary Light Yield.
The primary Light Yield: is due to the direct excitation and ionization produced by the SECS in the medium. The energy losses in the MACRO liquid scintillator is computed from the energy losses of protons in hydrogen and carbon [14]

$$
\begin{aligned}
& \left(\frac{d E}{d x}\right)_{S E C S}=\frac{1}{14}\left[2\left(\frac{d E}{d x}\right)_{H}+12\left(\frac{d E}{d x}\right)_{C}\right] \\
& =S P=\frac{S L \times S H}{S L+S H}
\end{aligned}
$$

where $S P$ is the stopping power of SECS, which reduces to $S L$ at low $\beta$ and to $S H$ at high $\beta$, so at very high $\beta$; the $S P$ stopping power coincides with the Bethe Bloch formula for electric energy losses.

1. For $Q=1$ the energy losses of SECS in hydrogen and carbon is computed from [11 adding an exponential factor due to the experimental data [12].

i) For $10^{-5}<\beta<5 \times 10^{-3}$ we obtained the following formula

$\left(\frac{d E}{d x}\right)_{S E C S}=C\left[1-\exp \left(\frac{\beta}{7 \times 10^{-4}}\right)^{2}\right] \frac{M e V}{c m}(20)$

where $C=1.3 \times 10^{5} \beta$.

ii) For $5 \times 10^{-3}<\beta<10^{-2}$ we used the following formula [12]

$S P=S P_{H}+S P_{C}=\left(\frac{d E}{d x}\right)_{S E C S}$

where

$S P_{H}=\frac{S L_{H} \times S H_{H}}{S L_{H}+S H_{H}}$

$S P_{C}=\frac{S L_{C} \times S H_{C}}{S L_{C}+S H_{C}}$

and

$S L=A_{1} E^{0.45}, \quad S H=A_{2} \operatorname{Ln}\left(1+\frac{A_{3}}{E}+A_{4} E\right)$

where $\left(A_{i=1,4}\right)$ are constants obtained from experimental data, and $E$ is the energy of a proton with velocity $\beta$.

2. For SECS with $Q=Z_{1} e$ the energy losses for $10^{-5}<\beta<10^{-2}$ are given by 13.

$\left(\frac{d E}{d x}\right)_{S E C S}=F\left(Z_{1}, Z_{2}\right)\left[1-\exp \left(-\frac{\beta}{7 \times 10^{-2}}\right)^{2}\right](25)$ 
where

$F\left(Z_{1}, Z_{2}\right)=\frac{8 \pi e^{2} a_{0} \beta}{\alpha} \frac{Z_{1}^{7 / 6} N_{e}}{\left(Z_{1}^{2 / 3}+Z_{2}^{2 / 3}\right)^{3 / 2}}$

where $Z_{2}$ is the atomic number of the target atom, $N_{e}$ the density of electrons and $\alpha$ is the fine structure constant. 14

The primary Light Yield of SECS is given by

$$
\left(\frac{d L}{d x}\right)_{S E C S}=A\left[\frac{1}{1+A B \frac{d E}{d x}}\right] \frac{d E}{d x}
$$

where $\frac{d E}{d x}$ is the energy loss of SECS; $A$ and $B$ are parameters depending only on the velocity of SECS.

The secondary Light Yield: we considered the elastic or quasielastic recoil of hydrogen and carbon nuclei. The light yield $L_{p}$ from a hydrogen or carbon nucleus of given initial energy $E$ is computed as

$L_{p}(E)=\int_{0}^{E} \frac{d L}{d x}(\epsilon) S_{\text {tot }}^{-1} d \epsilon$

where $S_{\text {tot }}$ is the sum of electronic and nuclear energy losses. The nuclear energy losses are given in ref. [15]. The secondary light yield is then

$$
\left(\frac{d L}{d x}\right)_{\text {secondary }}=N \int_{0}^{T_{m}} L_{p}(T) \frac{d \sigma}{d T} d T
$$

where $T_{m}$ is the maximum energy transferred and $\frac{d \sigma}{d T}$ is the differential scattering cross section, given in ref. [16].

In Fig. 3 is presented the light yield of SECS in MACRO liquid scintillator as function of the SECS velociy $\beta$.

\subsection{Energy losses of Q-balls type SECS in streamer tubes}

The composition of the gas in the MACRO limited streamer tubes is $73 \%$ helium, $27 \%$ n-pentane in volume [5]. The pressure is about one atmosphere and the resulting density is low (in comparison with the density of the other detectors): $\rho_{\text {gas }}=0.856 \mathrm{mg} / \mathrm{cm}^{3}$. The energy losses of MMs in the streamer tubes have been discussed in [5].

The ionization energy losses of SECS in the streamer tubes of the MACRO experiment for

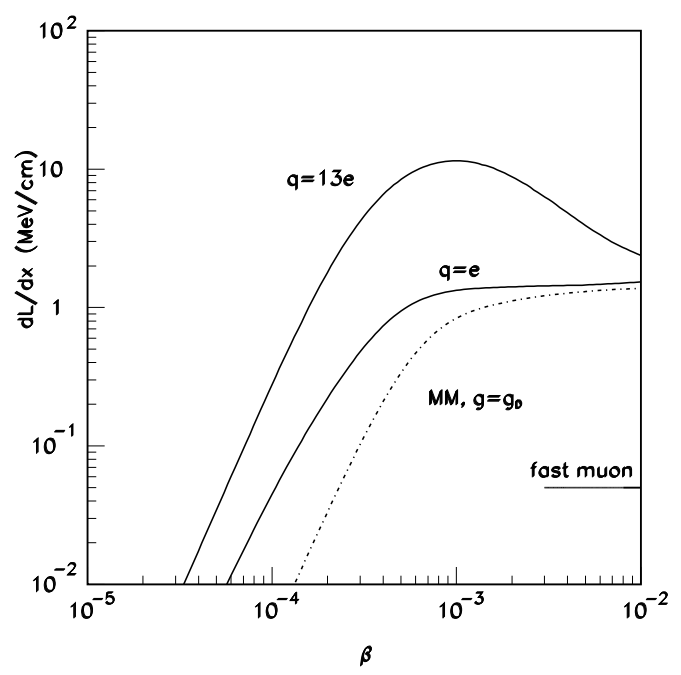

Figure 3. Light Yield of SECS in the MACRO liquid scintillator as function of the SECS velocity $\beta ; q$ is the net positive electric charge of the SECS core.

$10^{-3}<\beta<10^{-2}$ was computed with the same procedure used for scintillators, but using the density and the chemical composition of streamer tubes.

For $Q=13 e$ the energy losses are calculated from ref. 13] but we have omitted the exponential factor which takes into account the energy gap in organic scintillators.

The Drell effect does not occurs because SECS are not magnetically charged.

The threshold for ionizing n-pentane occur at $\beta \sim 10^{-3}$.

\subsection{Restricted Energy Losses of SECS in the Nuclear Track Detector CR39}

The quantity of interest for the CR39 nuclear track detector is the Restricted Energy Loss (REL), that is, the energy deposited within $\sim 100 \AA$ diameter from the track.

The REL in CR39 has already been computed for MMs of $g=g_{D}$ and $g=3 g_{D}$ and for dyons with $q=e, g=g_{D}$ [17. We have checked these 


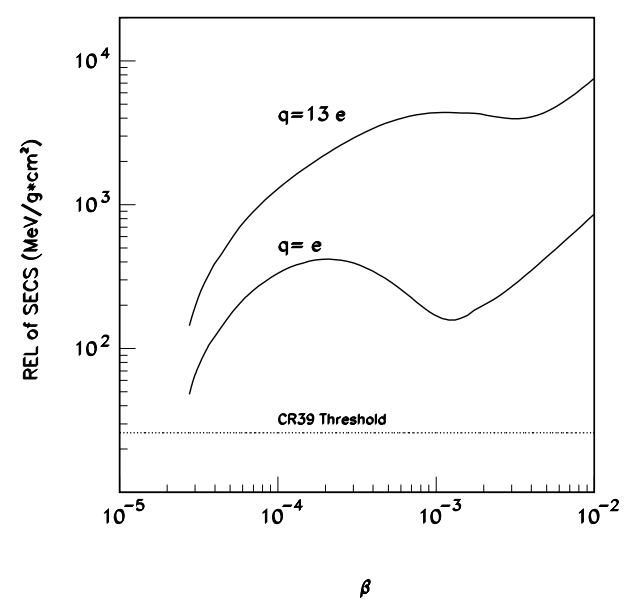

Figure 4. Restricted Energy Losses of SECS as function of velocity in the nuclear track detector CR39. The detection threshold for the MACRO CR39 is also shown ref. [5].

calculations and extended them to other cases of interest [5].

The chemical composition of CR39 is $\mathrm{C}_{12} \mathrm{H}_{18} \mathrm{O}_{7}$, and the density is $1.31 \mathrm{~g} / \mathrm{cm}^{3}$. For the computation of the REL only energy transfers to atoms above $12 \mathrm{eV}$ are considered, because it is estimated that $12 \mathrm{eV}$ are necessary to break the molecular bonds [14.

At low velocities $\left(3 \times 10^{-5}<\beta<10^{-2}\right)$ there are two contributions to REL: the ionization and the atomic recoil contributions.

The ionization contribution was computed with Ziegler's fit to the experimental data [1].

The atomic recoil contribution to REL was calculated using the interaction potential between an atom and a SECS which is equal to the electric potential [15] given by

$V(r)=\frac{Z_{1} Z_{2} e^{2}}{r} \phi(r)$

where $r$ is the distance between the core of SECS and the target atom, $e$ is the electric charge of the core, $Z_{2}$ is the atomic number of the target atom. The function $\phi(r)$ is the screening function given by 15 .

$\phi(r)=\sum_{1}^{3} C_{i} \exp \left[-\frac{b_{i} r}{a}\right]$

where $a$ is the screening length

$a=0.8853 \frac{a_{0}}{\left(Z_{1}^{\frac{1}{2}}+Z_{2}^{\frac{1}{2}}\right)^{\frac{2}{3}}}$

where $a_{0}$ is the Bohr radius; the coefficients are restricted such that

$\sum_{1}^{3} C_{i}=1$

Assuming the validity of this potential, we calculated the relation between the scattering angle $\theta$ and the impact parameter b. From this relation, the differential cross section $\sigma(\theta)$ is readily obtained as 14

$\sigma(\theta)=-(d b / d \theta) \cdot b / \sin \theta$

The relation between the transferred kinetic energy $\mathrm{K}$ and the scattering angle $\theta$ is given by the relation

$K=4 E_{\text {inc }} \sin ^{2}(\theta / 2)$

where $E_{\text {inc }}$ is the energy of the atom relative to the SECS in the center of mass system. The Restricted Energy Losses are finally obtained by integrating the transferred energies as

$-\frac{d E}{d x}=N \int \sigma(K) d K$

where $N$ is the number density of atoms in the medium, $\sigma(K)$ is the differential cross section as function of the transferred kinetic energy $\mathrm{K}$.

In Fig. 4 is presented the restricted energy losses of the Q-ball type SECS in the nuclear solide track detector CR39.

\section{CONCLUSION}

Supersymmetric generalizations of the Standard Model allow for stable non-topological solitons of Q-ball type which may be considered bags 
of squarks and sleptons and thus have non-zero baryon and lepton numbers, as well as the electric charge [1-3]. These solitons can be produced in the Early Universe, can affect the nucleosynthesis of light elements, and can lead to a variety of other cosmological consequences.

In this paper, we computed the energy losses of Q-balls of type SENS and SECS. Using these energy losses and a rough model of the earth's composition and density profiles, we have computed the geometrical acceptance of the MACRO detector for Q-balls type SECS with $v=250 \mathrm{~km} / \mathrm{s}$ as function of the Q-ball mass $M$. We have calculated the accessible region in the plane (mass, velocity) of SECS reaching MACRO from above and below.

We also presented a systematic analysis of the energy deposited in scintillators, streamer tubes and CR39 nuclear track detectors by SECS in forms useful for their detection. In particular we computed the light yield in scintillators, the ionization in streamer tubes and the REL in nuclear track detectors.

MACRO is sensitive to both SECS and SENS. A good upper flux limit may be obtained at the level of few times $10^{-16} \mathrm{~cm}^{-2} \mathrm{~s}^{-1} \mathrm{sr}^{-1}$.

Acknowledgements : I gratefully acknowledge Prof. G. Gaicomelli for his continous disponibility and for very useful critical discussions. This work was supported by ICTP and INFN grants.

\section{REFERENCES}

[1] T.D. Lee and Y. Pang, Phys. Rep. 221 (1992);

R. Ruffini and A. Bonnazola, Phys. Rev. 187 (1969) 1767;

J.D. Breit et al, Phys. Lett. B140 (1984) 329; M. Colpi et al, Phys. Rev. Lett. 57 (1986) 2485.

[2] S. Coleman, Nucl. Phys. B 262 (1985) 293.

[3] A. Kusenko, Phys. Lett. B 405 (1997) 108; Phys. Lett. B 404 (1997) 285; Phys. Lett. B 406 (1997) 26.

[4] A. Kusenko and M. Shaposhnikov, Phys. Lett. B 417 (1998) 99;

A. Kusenko et al., Phys. Rev. Lett. 80 (1998)
3185.

[5] J. Derkaoui et al, Astropart. Phys. 9 (1998) 173.

[6] E. Witten, Phys. Rev. D 30 (1984) 272;

A. De Rùjula and Glashow, Nature $\mathbf{3 1 2}$ (1984) 734 ;

E. Farhi and R.L. Jaffe, Phys. Rev. D 30 (1984) 2379.

[7] A. Kusenko, Phys. Rev. Lett. B 61 (1998) 2909;

J. Madsen, Phys. Lett. B 435 (1998) 125;

Phys. Lett. B 246 (1990) 135;

A.V. Olinto 'The Physics of Strange Matter', Proceedings of 'Relativistic Aspects of $\mathrm{Nu}$ clear Physics', Rio de Janiero, Brazil (1991); J. Madsen, astro-ph/9809032 (1998).

[8] V.A. Rubakov, Rep. Prog. Phys. 51 (1988) 189.

[9] M. Ouchrif, 'Energy losses of Q-balls' MACRO Int Memo, 7/99 (1999).

[10] T. Gherghetta, C. Kolda and S.P. Martin, Nucl. Phys. B468 (1996) 37;

G. Cleaver et al., hep-th/9711178 (1997).

[11] H. H. Andersen and J.F. Ziegler, Hydrogen stopping power and ranges in all elements, Pergamon Press (1977).

[12]D. J. Ficenec et al., Phys. Rev. D36 (1987) 311.

[13] J. Lindhard and M. Scharff, Phys. Rev. 124 (1961) 28.

[14] J. Derkaoui al., Astropart. Phys. 10 (1999) 339.

[15] W. D. Wilson, L. G. Haggmark and J. P. Biersack, Phys. Rev. B15 (1977) 2458.

[16]J. Lindhard et al, K. Dan. Vidensk. Selsk. Mat.-Fys. Med. 33 (1963) No. 14.

[17] T. W. Ruijgrok, J. A. Tjon and T. T. Wu, Phys. Lett. 129B ( S. Nakamura, Ph. D. Thesis, Search for supermassive relics by large area plastic track detectors, UT-ICEPP-8804, University of Tokyo (1988). 